\title{
MINIMAL DISCRETE ENERGY ON THE SPHERE
}

\author{
E. A. Rakhmanov, E. B. Saff, and Y. M. Zhou
}

\begin{abstract}
We investigate the energy of arrangements of $N$ points on the surface of a sphere in $\mathbf{R}^{3}$, interacting through a power law potential $V=$ $r^{\alpha},-2<\alpha<2$, where $r$ is Euclidean distance. For $\alpha=0$, we take $V=$ $\log (1 / r)$. An area-regular partitioning scheme of the sphere is devised for the purpose of obtaining bounds for the extremal (equilibrium) energy for such points. For $\alpha=0$, finer estimates are obtained for the dominant terms in the minimal energy by considering stereographical projections on the plane and analyzing certain logarithmic potentials. A general conjecture on the asymptotic form (as $N \rightarrow \infty$ ) of the extremal energy, along with its supporting numerical evidence, is presented. Also we introduce explicit sets of points, called "generalized spiral points", that yield good estimates for the extremal energy. At least for $N \leq 12,000$ these points provide a reasonable solution to a problem of M. Shub and S. Smale arising in complexity theory.
\end{abstract}

\section{Introduction}

Let $N \geq 2$ be a positive integer and $\omega_{N}=\left\{x_{1}, \ldots, x_{N}\right\}$ be a set of $N$ points on the unit sphere $S^{2}:=\left\{x \in \mathbf{R}^{3}:|x|=1\right\}$. We use $|x-y|$ to denote the Euclidean distance between two points $x, y \in S^{2}$. For each real $\alpha$, the $\alpha$-energy associated with $\omega_{N}$ is defined by

$$
E\left(\alpha, \omega_{N}\right):= \begin{cases}\sum_{1 \leq i<j \leq N} \log \frac{1}{\left|x_{i}-x_{j}\right|}, & \text { if } \alpha=0 \\ \sum_{1 \leq i<j \leq N}\left|x_{i}-x_{j}\right|^{\alpha}, & \text { if } \alpha \neq 0 .\end{cases}
$$

1991 Mathematics Subject Classification. Primary 52A40, 31C20, 41A60; Secondary 65Y20, 92E10, 92D20.

Received April 25, 1994.

The research of the first author was conducted while visiting the University of South Florida, Tampa.

The research of the second and third authors was supported, in part, by the U.S. National Science Foundation under grant DMS 920-3659.

The research done by the third author is in partial fulfillment of the Ph.D. at the University of South Florida. 
Our concern is with the extremal energy for $N$ points on the sphere:

$$
\mathcal{E}(\alpha, N):= \begin{cases}\inf _{\omega_{N} \subset S^{2}} E\left(\alpha, \omega_{N}\right), & \text { if } \alpha \leq 0 \\ \sup _{\omega_{N} \subset S^{2}} E\left(\alpha, \omega_{N}\right), & \text { if } \alpha>0 .\end{cases}
$$

The determination of $\mathcal{E}(\alpha, N)$ is an important and active research area (see the survey paper by Melnyk et al. [19]).

For example, determining the exact value of $\mathcal{E}(1, N)$, is, except for certain small values of $N$, a long-standing open problem in discrete geometry, which was initiated by L. Fejes Tóth [14]. Several authors, including Alexander [1], Stolarsky [26, 27] and Beck [2] have made significant contributions; see Stolarsky [27] for history.

The determination of $\mathcal{E}(-1, N)$ is called J. J. Thomson's Problem. Föppl [16], at the suggestion of Hilbert, made a rigorous examination of Thomson's arrangements; see L. L. Whyte [33] for history. Partly due to the recent discovery of carbon fullerenes $\left(\mathrm{C}_{60}, \mathrm{C}_{70}\right.$, etc.), see [9], [25], this problem has again attracted the attention of researchers in chemistry, physics and crystallography. There are hundreds of references to Thomson's Problem and its applications. Here we cite only a few recent ones (of more mathematical content): [11], [13], [17], [32], and [34].

We remark that for $\alpha=2$ it is easily verified that $\mathcal{E}(2, N)=N^{2}$ and that for $\alpha>2, N$ even, it is known that the points of a maximal distribution must all lie in two diametrically opposite points; see [4]. It is also interesting to note that as $\alpha \rightarrow-\infty$, the minimal discrete energy problem tends to the Best Packing Problem on the sphere (also known as Tammes' Problem), which asks for the largest spherical radius of $N$ identical spherical caps that can be packed onto the surface of the unit sphere; see Conway and Sloane [7], Coxeter [8] and Fejes Tóth [15].

For $\alpha=0$, a related problem was conveyed to the second author by S. Smale; namely the problem of finding explicit sets of points $\omega_{N}=$ $\left\{x_{1}, \ldots, x_{N}\right\} \subset S^{2}$ such that, for some constant $C_{0}$, we have

$$
E\left(0, \omega_{N}\right)-\mathcal{E}(0, N) \leq C_{0} \log N, \quad \forall N \geq 2 .
$$

Such points serve as good starting values for Newton's method (cf. Shub and Smale [22, 23, 24]).

Our goal is to provide bounds for the discrete extremal energy $\mathcal{E}(\alpha, N)$ when $-2<\alpha<2$ and to present a simple explicit formula for $N$ points on the sphere that yields good estimates for $\mathcal{E}(\alpha, N)$. The paper is organized as follows. In Section 2 we describe a general method for obtaining such bounds, provided that we can partition the sphere into $N$ parts of equal areas and small diameters. We present a scheme for this Partition 
Problem which yields nearly optimal rectangular zones. In Section 3, we develop an identity for $E\left(0, \omega_{N}\right)$ and use this to obtain upper bounds for $\mathcal{E}(0, N)$. We also improve on a lower bound for $\mathcal{E}(0, N)$ obtained by the late G. Wagner [29]. In Section 4, we formulate a general conjecture on the asymptotic form of $\mathcal{E}(\alpha, N)$ and discuss our numerical experiments that support the conjecture. In Section 5, we also describe a set of points, called generalized spiral points, that are extremely easy to construct and provide very good estimates for $\mathcal{E}(\alpha, N)$. Although for $\alpha=0$ these points do not appear to solve the Shub and Smale problem, they do yield good estimates; see (5.3).

\section{Energy Estimates and Partitions of the Sphere}

Our goal is to obtain bounds for the extremal energy by constructing suitable partitions of $S^{2}$. If $D \subset S^{2}$, we denote by $\stackrel{\circ}{D}$ the interior of $D$ with respect to $S^{2}$, and we denote by $A(D)$ the area of $D$ :

$$
A(D):=\int_{D} d \sigma(x)
$$

where $d \sigma$ is the surface area measure on $S^{2}$.

Definition 2.1. A collection $\mathcal{D}=\left\{D_{i}\right\}_{i=1}^{N}$ of $N$ closed subsets of $S^{2}$ is said to be an area-regular partition of $S^{2}$ into $N$ parts if

(i) $\bigcup_{i=1}^{N} D_{i}=S^{2}$;

(ii) $\stackrel{o}{D}_{i} \cap \stackrel{o}{D}_{j}=\emptyset, \quad i \neq j, \quad 1 \leq i, j \leq N$;

(iii) $A\left(\stackrel{o}{D}_{i}\right)=4 \pi / N, \quad i=1, \ldots, N$.

For $D \subset S^{2}$, the diameter $d(D)$ of $D$ is defined by

$$
d(D):=\sup \{|x-y|: x, y \in D\} .
$$

Later in this section we show how to construct area-regular partitions for which all regions $\stackrel{o}{D}_{i}$ have small diameters. The usefulness of such partitions is made clear by the following simple result.

Theorem 2.2. Let $K(r)$ be a lower semi-continuous decreasing function for $0<r \leq 2$ and suppose

$$
\beta(K):=\frac{1}{(4 \pi)^{2}} \iint_{S^{2} \times S^{2}} K(|x-y|) d \sigma(x) d \sigma(y)<\infty .
$$


If $\mathcal{D}=\left\{D_{i}\right\}_{i=1}^{N}$ is an area-regular partition of $S^{2}$ into $N$ parts, then there exist points $\left\{\hat{x}_{i}\right\}_{i=1}^{N}$ with $\hat{x}_{i} \in D_{i}, i=1, \ldots, N$, such that

$$
\sum_{1 \leq i \neq j \leq N} K\left(\left|\hat{x}_{i}-\hat{x}_{j}\right|\right) \leq N^{2} \beta(K)-\sum_{i=1}^{N} K\left(d\left(\stackrel{o}{D}_{i}\right)\right) .
$$

Remark. If $K$ is increasing and upper semi-continuous, then (2.2) is true with the inequality sign reversed.

Proof. Let $f\left(x_{1}, x_{2}, \ldots, x_{N}\right):=\sum_{i \neq j} K\left(\left|x_{i}-x_{j}\right|\right)$ and set

$$
\begin{gathered}
I:=\inf \left\{f\left(x_{1}, x_{2}, \ldots, x_{N}\right): x_{i} \in \stackrel{o}{D}, \quad i=1, \ldots, N\right\}, \\
d \sigma^{*}:=\frac{N}{4 \pi} d \sigma, \quad d \sigma_{i}^{*}:=\left.d \sigma^{*}\right|_{D_{i}} ^{o}, \quad i=1, \ldots, N,
\end{gathered}
$$

so that $\sigma_{i}^{*}(\stackrel{\circ}{D} j)=\delta_{i j}$. Then integrating the inequality $I \leq f\left(x_{1}, \ldots, x_{N}\right)$ with respect to $d \sigma_{1}^{*}\left(x_{1}\right) d \sigma_{2}^{*}\left(x_{2}\right) \cdots d \sigma_{N}^{*}\left(x_{N}\right)$ we get

$$
\begin{aligned}
I & \leq \int \cdots \int \sum_{i \neq j} K\left(\left|x_{i}-x_{j}\right|\right) d \sigma_{1}^{*}\left(x_{1}\right) d \sigma_{2}^{*}\left(x_{2}\right) \cdots d \sigma_{N}^{*}\left(x_{N}\right) \\
& =\sum_{i \neq j} \iint_{\substack{o \\
D_{i} D_{j}}} K(|x-y|) d \sigma_{i}^{*}(x) d \sigma_{j}^{*}(y) \\
& =\iint K(|x-y|) d \sigma^{*}(x) d \sigma^{*}(y)-\sum_{\substack { i=1 \\
\begin{subarray}{c}{o \\
D_{i} \times D_{i}{ i = 1 \\
\begin{subarray} { c } { o \\
D _ { i } \times D _ { i } } }\end{subarray}} \iint_{\substack{o \\
D_{i}}} K(|x-y|) d \sigma_{i}^{*}(x) d \sigma_{i}^{*}(y),
\end{aligned}
$$

where in the last equality we used the fact that the boundary of each $D_{i}$ has area measure zero. Since $K$ is decreasing, we obtain from (2.5) and (2.1) that

$$
I \leq N^{2} \beta(K)-\sum_{i=1}^{N} K\left(d\left(\stackrel{o}{D}_{i}\right)\right),
$$

which yields (2.2) for suitable $\hat{x}_{i} \in D_{i}$.

We remark that the above proof is similar to that of Lemma 4 in [1] and that Theorem 2.2 immediately extends to partitions of the unit sphere $S^{n-1} \subset \mathbf{R}^{n}$.

Theorem 2.3. For any $N \geq 2$, there exists an area-regular partition of $S^{2}$ into $N$ parts with the diameter of each part $\leq 7 / \sqrt{N}$. 
Remark. The existence of an absolute constant $C$ such that, for all $N \geq 2$, the sphere can be partitioned into $N$ parts of equal area with the diameter of each part $\leq C / \sqrt{N}$ is well known and is used in Alexander [1], Bourgain and Lindenstrauss [5, 6], Beck and Chen [3]. Necessarily, $C \geq 4$ since among all the subsets of $S^{2}$ with fixed area $4 \pi / N$, the spherical cap has minimal diameter, which equals $4 \sqrt{N-1} / N$. For large $N$ the constant 7 in Theorem 2.3 can be improved; for example, if $N \geq 3100$, we can find an area-regular partition with each piece having diameter $\leq 6 / \sqrt{N}$. Furthermore, if we only insist that most of the parts have small diameters, then it is possible to partition the sphere into nearly spherical square pieces, as described in the following result.

Theorem 2.4. Given $0<\varepsilon \leq 1$, there exist $k_{0}, N_{0}$ such that if $N \geq N_{0}$, an area-regular partition $\mathcal{D}=\left\{D_{j}\right\}_{j=1}^{N}$ of $S^{2}$ exists satisfying

$$
d\left(D_{j}\right) \leq \begin{cases}2 \sqrt{2 \pi}(1+\varepsilon) / \sqrt{N}, & \text { if } k_{0}+1 \leq j \leq N-k_{0} \\ 2 \sqrt{k_{0}} / \sqrt{N} & \text { otherwise }\end{cases}
$$

Since the proofs of Theorems 2.3 and 2.4 are similar, we sketch only the proof of Theorem 2.4. For convenience, we will call a sequence $\left\{y_{k}\right\}_{k=1}^{n}$ of real numbers symmetric if $y_{k}=y_{n-k+1}$ for all $1 \leq k \leq n$, and we make use of following lemma, which can be proved by mathematical induction.

Lemma 2.5. If $n$ is an odd positive integer, and $\left\{y_{i}\right\}_{i=1}^{n}$ is a symmetric sequence of real numbers with the property that $\sum_{i=1}^{n} y_{i}$ is an integer, then there exists a symmetric sequence of integers $\left\{m_{i}\right\}_{i=1}^{n}$ such that

$$
\begin{aligned}
& \text { i) } \sum_{i=1}^{n} m_{i}=\sum_{i=1}^{n} y_{i} ; \\
& \text { ii) }\left|y_{1}-m_{1}\right|=\left|y_{n}-m_{n}\right| \leq \frac{1}{2} ; \quad\left|y_{i}-m_{i}\right| \leq 1, \quad i=2, \ldots, n-1 ; \\
& \text { iii) }\left|\sum_{i=1}^{k}\left(y_{i}-m_{i}\right)\right| \leq \frac{1}{2}, \quad \forall k=1,2, \ldots, n .
\end{aligned}
$$

Let $(\theta, \phi), \quad 0 \leq \theta \leq \pi, \quad 0 \leq \phi \leq 2 \pi$, denote the spherical coordinates on $S^{2}$. For any partition of the integer $N$, say $\gamma=\left(m_{1}, \ldots, m_{n}\right)$, we associate an area-regular partition $\mathcal{D}=\left\{D_{k, j}\right\}$ of the sphere as follows:

$$
D_{k, j}:=\left[\theta_{k-1}, \theta_{k}\right] \times\left[\frac{2 \pi(j-1)}{m_{k}}, \frac{2 \pi j}{m_{k}}\right], \quad j=1, \ldots, m_{k}, \quad k=1, \ldots, n,
$$

where $\theta_{k}:=\arccos \left(1-(2 / N) \sum_{i=1}^{k} m_{i}\right), \quad k \geq 1$, and $\theta_{0}:=0$. We call this area-regular partition a $\gamma$-partition. 
Proof of Theorem 2.4. Given $0<\varepsilon \leq 1$, define $\eta:=1-(1+\varepsilon)^{-1 / 2}$,

$$
\begin{gathered}
k_{0}:=\left[\frac{(\sqrt{\pi}+\eta)^{2}}{2 \eta^{2}}\right]+1, \quad N_{0}:=\left[\frac{16 \pi k_{0}}{\eta^{2}}\right]+1, \\
n:=\{\text { the greatest odd integer } \leq \sqrt{\pi N} / 2\}, \\
\theta_{0}:=\arccos \left(1-\frac{2 k_{0}}{N}\right) ; \quad \Delta \theta:=\frac{\pi-2 \theta_{0}}{n}, \\
\theta_{k}^{\prime}=\theta_{0}+k \cdot \Delta \theta, \quad 0 \leq k \leq n, \quad \theta_{n+1}:=\theta_{n+1}^{\prime}:=\pi ; \\
y_{k}=\frac{N}{2}\left(\cos \theta_{k-1}^{\prime}-\cos \theta_{k}^{\prime}\right), \quad k=1,2, \ldots, n .
\end{gathered}
$$

Then $\sum_{k=1}^{n} y_{k}=N-2 k_{0}$ and $\left\{y_{k}\right\}_{k=1}^{n}$ is symmetric. Let $\left\{m_{k}\right\}_{k=1}^{n}$ be the symmetric sequence of integers that satisfies (i), (ii) and (iii) of Lemma 2.5. Then the $\gamma$-partition corresponding to $\gamma=\left(k_{0}, m_{1}, \ldots, m_{n}, k_{0}\right)$ can be shown to satisfy the conclusion of the theorem. The details of this verification will appear in [35].

Remark. Without the area-regular requirement, the sphere can be partitioned into $N$ parts with the diameter of each part less than

$$
d_{N}:=\frac{4}{\sqrt{N}} \cdot \sqrt{\frac{2 \pi}{\sqrt{27}}}\left(1+\varepsilon_{N}\right), \quad \varepsilon_{N}>0, \quad \varepsilon_{N} \rightarrow 0 \text { as } N \rightarrow \infty
$$

Indeed, as a consequence of a result due to van der Waerden [28], the sphere can be covered by $N$ identical (overlapping) spherical caps of diameter $d_{N}$ given in (2.6). Then the Dirichlet cells corresponding to the centers of these $N$ caps form a partition of the sphere into $N$ parts with each part having diameter $\leq d_{N}$. Although this does not yield an area-regular partition, it is tempting to conjecture that for

$$
\gamma_{N}:=\inf \left\{\max _{1 \leq j \leq N} d\left(D_{j}\right):\left\{D_{j}\right\}_{j=1}^{N} \text { is an area-regular partition of } S^{2}\right\},
$$

we have $\lim _{N \rightarrow \infty} \sqrt{N} \gamma_{N}=4 \sqrt{2 \pi / \sqrt{27}}=4.39854 \ldots$.

Using the fact that a zonal cut of the sphere has an area equal to $2 \pi$ times the height of the cut, it is easy to verify that for the kernel $K(r)=r^{\alpha},-2<\alpha<2, \alpha \neq 0$, we have

$$
\frac{1}{4 \pi} \int_{S^{2}}|x-y|^{\alpha} d \sigma(x)=\frac{2^{\alpha+1}}{2+\alpha}, \quad y \in S^{2}
$$

hence $\beta(K)=2^{\alpha+1} /(2+\alpha)$ (cf. (2.1)), and so from Theorems 2.2 and 2.4 we deduce 
Corollary 2.6. Given $-2<\alpha<2, \alpha \neq 0$, and $\varepsilon>0$, there exists an $N_{0}=N_{0}(\varepsilon, \alpha)$ such that for any $N \geq N_{0}$,

$$
\begin{aligned}
& \mathcal{E}(\alpha, N) \leq \frac{2^{\alpha}}{2+\alpha} N^{2}-\frac{1}{2}(2 \sqrt{2 \pi})^{\alpha}(1-\varepsilon) N^{1-\alpha / 2}, \quad \text { if }-2<\alpha<0, \\
& \mathcal{E}(\alpha, N) \geq \frac{2^{\alpha}}{2+\alpha} N^{2}-\frac{1}{2}(2 \sqrt{2 \pi})^{\alpha}(1+\varepsilon) N^{1-\alpha / 2}, \quad \text { if } 0<\alpha<2 .
\end{aligned}
$$

See Section 4 for a discussion of bounds in the opposite directions for $\mathcal{E}(\alpha, N)$.

\section{Logarithmic Equilibrium Points on the Sphere}

Here we consider the case $\alpha=0$ in more detail. Since $e^{-E\left(0, \omega_{N}\right)}=$ $\prod_{1 \leq i<j \leq N}\left|x_{i}-x_{j}\right|$, the minimization of the discrete energy $E\left(0, \omega_{N}\right)$ is the same as the maximization of the Vandermonde. Such extremal points are called logarithmic equilibrium points on $S^{2}$.

Elkies (cf. [18, p. 150]) describes a general method for obtaining lower bounds for minimal logarithmic energy that is valid over more general Riemann surfaces. His method shows, for example, that in the case of the sphere we have

$$
\mathcal{E}(0, N) \geq-\frac{1}{4} \log \left(\frac{4}{e}\right) N^{2}-\frac{1}{4} N \log N+O(N) .
$$

Our main concern is to obtain explicit estimates for the $O(N)$ term.

G. Wagner [29] proved that, for any $\omega_{N}=\left\{x_{1}, x_{2}, \ldots, x_{N}\right\}$ on $S^{2}$,

$$
\prod_{i<j}\left|x_{i}-x_{j}\right| \leq 2^{N(N-1) / 2}\left[\prod_{k=1}^{N-1} \frac{k !}{k^{k}}\right]^{1 / 2} .
$$

Here we present a modification of Wagner's proof that enables us to improve (3.1). The proof lends itself to further improvement and may ultimately lead to an asymptotically sharp upper bound for $\prod_{i<j}\left|x_{i}-x_{j}\right|$.

Theorem 3.1. For any $\omega_{N}=\left\{x_{1}, x_{2}, \ldots x_{N}\right\}$ on $S^{2}$, there holds

$$
\begin{aligned}
\prod_{1 \leq i<j \leq N}\left|x_{i}-x_{j}\right| & \leq 2^{N(N-1) / 2}\left[\prod_{k=1}^{N-1} \frac{k !}{k^{k}}\right]^{1 / 2}\left(1-e^{-a}+\varepsilon_{N}\right)^{b N / 4} \\
& \leq(4 / e)^{N^{2} / 4} N^{N / 4}(\pi / 2)^{N / 4}\left(1-e^{-a}+\varepsilon_{N}\right)^{b N / 4},
\end{aligned}
$$

where

$$
a:=\frac{2 \sqrt{2 \pi}}{\sqrt{27}}(\sqrt{2 \pi}+\sqrt{2 \pi+\sqrt{27}}), \quad b:=\frac{\sqrt{2 \pi+\sqrt{27}}-\sqrt{2 \pi}}{\sqrt{2 \pi+\sqrt{27}}+\sqrt{2 \pi}},
$$


and $\varepsilon_{N}>0, \varepsilon_{N} \rightarrow 0$ as $N \rightarrow \infty$. Thus, defining $C_{N}$ by

$$
\mathcal{E}(0, N)=-\frac{1}{4} \log \left(\frac{4}{e}\right) N^{2}-\frac{1}{4} N \log N+C_{N} N,
$$

there holds

(3.4) $\liminf _{N \rightarrow \infty} C_{N} \geq-\frac{1}{4} \log \left(\frac{\pi}{2}(1-\exp (-a))^{b}\right)=-0.112768770 \ldots$

We remark that the coefficient $-(1 / 4) \log (4 / e)$ of the $N^{2}$ term in (3.3) is just half of the average of $K(|x-y|)=\log |x-y|^{-1}$ over $S^{2} \times S^{2}$ as in Theorem 2.2.

Proof of Theorem 3.1. Let $\mathcal{S}: S^{2} \rightarrow \mathbf{C}$ be the stereographical projection of $S^{2}$ onto the complex plane $\mathbf{C}$. Let $z_{i}=\mathcal{S}\left(x_{i}\right), \quad i=1,2, \ldots, N$. Then we have

$$
\begin{aligned}
\prod_{i<j}\left|x_{i}-x_{j}\right| & =\prod_{i<j} \frac{2\left|z_{i}-z_{j}\right|}{\sqrt{\left(1+\left|z_{i}\right|^{2}\right)\left(1+\left|z_{j}\right|^{2}\right)}} \\
& =\frac{2^{N(N-1) / 2}}{\prod_{k=1}^{N}\left(1+\left|z_{k}\right|^{2}\right)^{(N-1) / 2}} \prod_{i<j}\left|z_{i}-z_{j}\right|,
\end{aligned}
$$

and so

$$
\prod_{i<j}\left|x_{i}-x_{j}\right|=2^{N(N-1) / 2} \prod_{k=1}^{N-1}\left(\begin{array}{c}
N-1 \\
k
\end{array}\right)^{-1 / 2} \cdot\left|\operatorname{det}\left(\boldsymbol{\xi}_{\mathbf{1}}, \boldsymbol{\xi}_{\mathbf{2}}, \ldots, \boldsymbol{\xi}_{\boldsymbol{N}}\right)\right|,
$$

where

$$
\begin{array}{r}
\xi_{k}:=\frac{1}{\left(1+\left|z_{k}\right|^{2}\right)^{(N-1) / 2}}\left(\sqrt{\left(\begin{array}{c}
N-1 \\
0
\end{array}\right)}, \sqrt{\left(\begin{array}{c}
N-1 \\
1
\end{array}\right)} z_{k}, \ldots, \sqrt{\left(\begin{array}{l}
N-1 \\
N-1
\end{array}\right)} z_{k}^{N-1}\right), \\
k=1, \ldots, N .
\end{array}
$$

Using the fact that

$$
\prod_{k=0}^{N-1}\left(\begin{array}{c}
N-1 \\
k
\end{array}\right)=\prod_{k=1}^{N-1} \frac{k^{k}}{k !}
$$

we get

$$
\prod_{i<j}\left|x_{i}-x_{j}\right|=2^{N(N-1) / 2}\left[\prod_{k=1}^{N-1} \frac{k !}{k^{k}}\right]^{1 / 2} \cdot\left|\operatorname{det}\left(\boldsymbol{\xi}_{\mathbf{1}}, \boldsymbol{\xi}_{\mathbf{2}}, \ldots, \boldsymbol{\xi}_{N}\right)\right| .
$$

Notice that each $\boldsymbol{\xi}_{\boldsymbol{k}}$ regarded as a vector in $\mathbf{C}^{N}$ has $l_{2}$-norm equal to one; consequently, from Hadamard's inequality, we deduce (3.1). 
To improve the estimate (3.1), we shall (for many indices $i$ ) subtract from the vector $\boldsymbol{\xi}_{\boldsymbol{i}}$ its projection on a nearby neighbor $\boldsymbol{\xi}_{\boldsymbol{j}}$. For this purpose, we recall from (2.6) that, for $\lambda>1$ (to be chosen later), we can partition the sphere into $[N / \lambda]$ parts with the diameter of each part less than

$d_{N, \lambda}:=(4 \sqrt{\lambda} / \sqrt{N}) \cdot \sqrt{2 \pi / \sqrt{27}}\left(1+\varepsilon_{N}\right), \quad \varepsilon_{N}>0, \varepsilon_{N} \rightarrow 0$ as $N \rightarrow \infty$.

Let us now consider a selection process in which we pair off points $\left\{x_{i}, x_{j}\right\}$, $i \neq j$, if $x_{i}, x_{j}$ belong to the same part of this partition. Since there are $[N / \lambda]$ parts, it can be seen that there exist at least $(1-1 / \lambda) N / 2$ pairwise disjoint sets of points $\left\{x_{i}, x_{j}\right\}_{i \neq j}$ with $\left|x_{i}-x_{j}\right| \leq d_{N, \lambda}$. For these sets, the corresponding vector sets $\left\{\boldsymbol{\xi}_{\boldsymbol{i}}, \boldsymbol{\xi}_{\boldsymbol{j}}\right\}$ satisfy

$$
\left|\left\langle\boldsymbol{\xi}_{\boldsymbol{i}}, \boldsymbol{\xi}_{\boldsymbol{j}}\right\rangle\right|^{2}=\left(1-\frac{\left|x_{i}-x_{j}\right|^{2}}{4}\right)^{N-1} \geq\left(1-\frac{d_{N, \lambda}^{2}}{4}\right)^{N-1} .
$$

Since $d_{N, \lambda}^{2} / 4=(8 \pi \lambda / \sqrt{27})\left(1+\varepsilon_{N}\right)^{2} / N$ and $(1+x)^{N-1} \geq \exp ((N-1) x /(1+$ $x)$ ) for any $x>-1, x \neq 0$, inequality (3.7) implies that

$$
\begin{aligned}
\left|\left\langle\boldsymbol{\xi}_{\boldsymbol{i}}, \boldsymbol{\xi}_{\boldsymbol{j}}\right\rangle\right|^{2} & \geq \exp \left(-\frac{(8 \pi \lambda / \sqrt{27})\left(1+\varepsilon_{N}\right)^{2}(N-1)}{N-(8 \pi \lambda / \sqrt{27})\left(1+\varepsilon_{N}\right)^{2}}\right) \\
& \geq \exp (-8 \pi \lambda / \sqrt{27})-\hat{\varepsilon}_{N},
\end{aligned}
$$

where $\hat{\varepsilon}_{N}>0, \hat{\varepsilon}_{N} \rightarrow 0$ as $N \rightarrow \infty$. Hence,

$$
\left|\boldsymbol{\xi}_{\boldsymbol{i}}-\left\langle\boldsymbol{\xi}_{\boldsymbol{i}}, \boldsymbol{\xi}_{\boldsymbol{j}}\right\rangle \boldsymbol{\xi}_{\boldsymbol{j}}\right|=\sqrt{1-\left|\left\langle\boldsymbol{\xi}_{\boldsymbol{i}}, \boldsymbol{\xi}_{\boldsymbol{j}}\right\rangle\right|^{2}} \leq\left(1-\exp (-8 \pi \lambda / \sqrt{27})+\hat{\varepsilon}_{N}\right)^{1 / 2} .
$$

Subtracting the projections for the above pairs and applying Hadamard's inequality, we deduce from (3.8) and (3.6) that

$$
\begin{aligned}
& \prod_{i<j}\left|x_{i}-x_{j}\right| \\
\leq & 2^{N(N-1) / 2}\left[\prod_{k=1}^{N-1} \frac{k !}{k^{k}}\right]^{1 / 2} \cdot\left(1-\exp (-8 \pi \lambda / \sqrt{27})+\hat{\varepsilon}_{N}\right)^{(1-1 / \lambda) N / 4} .
\end{aligned}
$$

Next we want to minimize the upper bound in (3.9) with respect to $\lambda$. Since $\exp (-8 \pi \lambda / \sqrt{27})$ is very small, we have

$$
\log \left(1-\exp (-8 \pi \lambda / \sqrt{27})+\hat{\varepsilon}_{N}\right)^{(1-1 / \lambda)} \approx-(1-1 / \lambda) \exp (-8 \pi \lambda / \sqrt{27}),
$$


and the minimization of the last term gives $\lambda=(\sqrt{2 \pi}+\sqrt{2 \pi+\sqrt{27}}) / 2 \sqrt{2 \pi}$. Plugging this value for $\lambda$ into (3.9) we obtain the first part of (3.2). Then, the second part of (3.2) and (3.4) follow (after some calculations) by Stirling's formula. (We remark that the explicit choice of $\lambda$ in the preceding proof is not optimal; a slightly smaller choice for $\lambda$ leads to a slight improvement in the upper bound in (3.2).)

In the opposite direction, we have

Theorem 3.2. With $C_{N}$ defined as in Theorem 3.1,

$$
\limsup _{N \rightarrow \infty} C_{N} \leq-\frac{1}{4} \log \frac{\pi \sqrt{3}}{2}-\frac{\pi}{8 \sqrt{3}}=-0.0234973 \ldots
$$

The proof of Theorem 3.2 requires much more effort. Here we present only a sketch; details will appear in [21]. The idea is to construct an explicit set of points whose energy is easy to estimate yet provides a good upper bound for $C_{N}$. For this purpose it is convenient to first define points in the plane and then take their projections on $S^{2}$.

Proof. Given $N$ and any partition of $N$, say $\left\{m_{1}, m_{2}, \ldots, m_{n}\right\}$, where $\sum_{k=1}^{n} m_{k}=N$ and $m_{k}>0, k=1, \ldots, N$, let $\alpha_{k} \in[0,1), k=1, \ldots, n$. Define, for $1 \leq k \leq n$,

$$
\begin{array}{ll}
\xi_{k}:=1-\frac{1}{N}\left(\sum_{j=1}^{k-1} m_{j}+\frac{m_{k}}{2}\right), & r_{k}:=\sqrt{\frac{1}{\xi_{k}}-1} \\
z_{k, j}:=r_{k} \exp \left(\frac{2 \pi i}{m_{k}}\left(j+\alpha_{k}\right)\right), & j=1,2, \ldots, m_{k} .
\end{array}
$$

Let $d \sigma^{*}:=(N / 4 \pi) \cdot d \sigma$, where $d \sigma$ is the surface area measure on $S^{2}$, and set $d \tau:=\sum_{k=1}^{n} d \tau_{k}$, where $d \tau_{k}:=m_{k}(d \varphi / 2 \pi)$ with $d \varphi$ denoting angular measure on the circle $|z|=r_{k}$. As before, we let $\mathcal{S}$ denote the stereographical projection and we consider the point set

$$
\omega_{N}:=\left\{\mathcal{S}^{-1}\left(z_{k, j}\right): 1 \leq j \leq m_{k}, 1 \leq k \leq n\right\}
$$

Then we have the following representation. 
Lemma 3.3. Let $D_{k}:=\left\{z \in \mathbf{C}: r_{k-1} \leq|z| \leq r_{k}\right\}, r_{0}:=0$ and let $d \lambda=d \sigma^{*}\left(\mathcal{S}^{-1}\right)$ and $d \lambda_{k}:=\left.d \lambda\right|_{D_{k}}$. Then

$$
\begin{aligned}
&-E\left(0, \omega_{N}\right)= \frac{1}{4} \log \left(\frac{4}{e}\right) N^{2}+\frac{1}{2} \sum_{k=1}^{n} m_{k} \log \frac{m_{k}}{2 \sqrt{\xi_{k}\left(1-\xi_{k}\right)}} \\
&+ \frac{1}{2} \sum_{k=1}^{n} \int V^{\lambda_{k}-\tau_{k}} d(\lambda+\tau) \\
&+\sum_{1 \leq l<k \leq n} d_{l, k} \log \left|1-\left(\frac{r_{l}}{r_{k}}\right)^{m_{l} m_{k} / d_{l, k}} \exp \left(2 \pi i \frac{\alpha_{l} m_{k}-\alpha_{k} m_{l}}{d_{l, k}}\right)\right|,
\end{aligned}
$$

where $d_{l, k}$ is the greatest common divisor of $m_{l}$ and $m_{k}$ and $V^{\lambda_{k}-\tau_{k}}(z)$, $z \in \mathbf{C}$, is the logarithmic potential of $\lambda_{k}-\tau_{k}$, i.e.,

$$
V^{\lambda_{k}-\tau_{k}}(z):=\int \log \frac{1}{|x-z|} d\left(\lambda_{k}-\tau_{k}\right)(x)
$$

Remark. The term in (3.11) involving the integral of the potential can be represented as

$$
\begin{aligned}
\sum_{k=1}^{n} \frac{1}{2} \int V^{\lambda_{k}-\tau_{k}} d(\lambda+\tau)=\frac{1}{24 N} \sum_{k=1}^{n} m_{k}^{3}\left(\frac{1}{\xi_{k}}+\frac{1}{1-\xi_{k}}\right) & \\
+O & {\left[\frac{1}{N^{2}} \sum_{k=1}^{n} m_{k}^{4}\left(\frac{1}{\xi_{k}^{2}}+\frac{1}{\left(1-\xi_{k}\right)^{2}}\right)\right] . }
\end{aligned}
$$

Notice that the last term in (3.11) is the only term that involves the "angular adjustments" $\alpha_{k}$. If we integrate this term with respect to $d \alpha_{1} d \alpha_{2} \cdots d \alpha_{n}$ for $0 \leq \alpha_{k} \leq 1$, we get zero, which means there is at least one set of angular adjustments with the property that the last term in (3.11) vanishes. With this choice of $\alpha_{k}$ 's we select $n$ and $\left\{m_{k}\right\}_{k=1}^{n}$ exactly as in the proof of Theorem 2.4. After substantial calculations, it can be shown that (3.10) is true.

The next result shows that logarithmic equilibrium points are wellseparated on the sphere; its proof will appear in [20].

Theorem 3.4. If $\left\{x_{i}^{(N)}\right\}_{i=1}^{N} \subset S^{2}$ maximizes the product $\prod_{1 \leq i<j \leq N}\left|x_{i}-x_{j}\right|$ for $\left\{x_{i}\right\}_{1}^{N} \subset S^{2}$, then

$$
\left|x_{i}^{(N)}-x_{j}^{(N)}\right| \geq \frac{3}{5} \frac{1}{\sqrt{N}}, \quad \text { for } i \neq j .
$$


Elkies [12] also has observed that in the logarithmic equilibrium case $(\alpha=0)$, the order of separation is $O(1 / \sqrt{N})$. For $\alpha=-1$, Dahlberg has proved in [10] the well-separatedness of the minimum energy points.

\section{Conjectures for Asymptotics of $\mathcal{E}(\alpha, N)$}

Several conjectures already exist concerning the asymptotic behavior of $\mathcal{E}(\alpha, N)$ for special values of $\alpha$ : see Alexander [1], Stolarsky [26, 27] and Beck [2] for $\alpha=1$; Glasser [17] and Erber [13] for $\alpha=-1$. Here we formulate a general conjecture for $\mathcal{E}(\alpha, N)$ and discuss our numerical experiments for the cases $N \leq 200, \alpha=0, \pm 1$, that support it.

Conjecture 4.1. For $-2<\alpha<2$ there exist absolute constants $B_{\alpha}, C_{\alpha}$, depending only on $\alpha$, such that

$$
\begin{aligned}
& \text { (4.1) } \mathcal{E}(\alpha, N) \\
& = \begin{cases}-\frac{1}{4} \log \left(\frac{4}{e}\right) N^{2}-\frac{1}{4} N \log N+B_{\alpha} N+C_{\alpha} \log N+O(1) & \text { if } \alpha=0, \\
\frac{2^{\alpha}}{2+\alpha} N^{2}+B_{\alpha} N^{1-\alpha / 2}+C_{\alpha} N^{-\alpha / 2}+O\left(N^{-1-\alpha / 2}\right) & \text { if } \alpha \neq 0 .\end{cases}
\end{aligned}
$$

Assuming the validity of this conjecture, it follows from (3.4) and (3.10) that, for $\alpha=0$,

$$
-0.1127688 \leq B_{0} \leq-0.0234973 .
$$

For $\alpha \neq 0$, Corollary 2.6 yields an upper bound for $B_{\alpha}$ if $-2<\alpha<0$ and a lower bound for $B_{\alpha}$ if $0<\alpha<2$. We can also obtain (after some calculations) a lower bound for $B_{\alpha}$ when $-2<\alpha<0$ using the results of [30]. Combining these estimates we get

$$
\begin{gathered}
-\frac{5+2 \alpha}{4+2 \alpha} \leq B_{\alpha} \leq-\frac{1}{2}(2 \sqrt{2 \pi})^{\alpha}, \quad \text { if }-2<\alpha<0 \\
-\frac{1}{2}(2 \sqrt{2 \pi})^{\alpha} \leq B_{\alpha}<0, \quad \text { if } 0<\alpha<2 .
\end{gathered}
$$

The upper bound in the last inequality is proved in [31].

We did massive high precision computer experiments to find the extremal points and determine $\mathcal{E}(\alpha, N)$ for $\alpha=0, \pm 1$ and $N \leq 200$. An ad hoc algorithm was designed for the case $\alpha=0$. We also tried several known algorithms and found that the quasi-Newton algorithm works quite well for general $\alpha$. Because of the presence of local extrema, care had to be taken to ensure that the extrema we found were indeed global extrema. For the cases we investigated, the extremal configurations on the sphere 
demonstrate a rich set of symmetries and principles. We also observed that all the local extrema have very close energies.

Fitting the conjectured formulas $(4.1)^{\dagger}$ to the data obtained for $\alpha=$ $0, \pm 1$, by minimizing the absolute $l_{1}$-deviation (for $N \leq 200$ ), leads to the following formulas for the approximations $f(\alpha, N)$ to the actual values of $\mathcal{E}(\alpha, N)$ :

$$
\begin{gathered}
f(0, N)=-\frac{1}{4} \log \left(\frac{4}{e}\right) N^{2}-\frac{1}{4} N \log N-0.026422 N+0.13822 \\
f(1, N)=\frac{2}{3} N^{2}-0.40096 N^{1 / 2}-0.188 N^{-1 / 2}
\end{gathered}
$$

$$
f(-1, N)=\frac{N^{2}}{2}-0.55230 N^{3 / 2}+0.0689 N^{1 / 2}
$$

In Figure 1 we plot the difference $\mathcal{E}(0, N)-f(0, N)$. Notice from the enlarged scale that the fit is quite good. The graphs for $\alpha= \pm 1$ are similar.

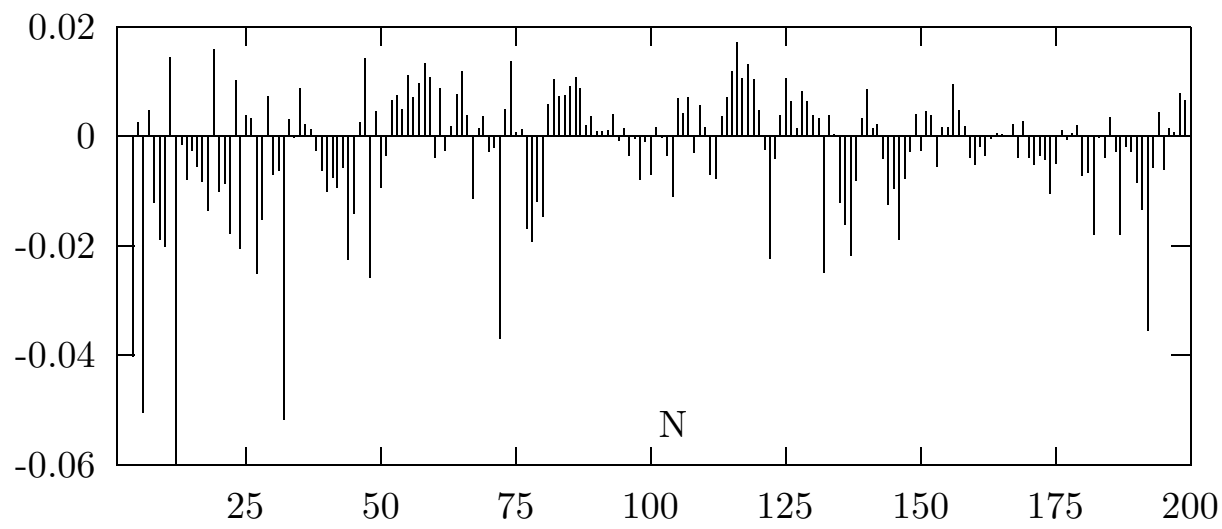

Figure 1. Error in Approximating Extremal Logarithmic Energy

\footnotetext{
${ }^{\dagger}$ For the case $\alpha=0$, the $\log N$ term did not appear to be significant and was ignored in the fitting algorithm.
} 


\section{Generalized spiral points}

From numerical experiments, it appears that the logarithmic equilibrium points try to distribute themselves over a nearly regular spherical hexagonal net. We devised a simple scheme for imitating this behavior for any given $N$. To describe these points we use the spherical coordinates $(\theta, \phi), 0 \leq \theta \leq \pi, 0 \leq \phi \leq 2 \pi$. Let

$$
\begin{gathered}
h_{k}:=-1+2(k-1) /(N-1), \quad 1 \leq k \leq N ; \quad \theta_{k}:=\arccos \left(h_{k}\right) ; \\
\phi_{1}:=\phi_{N}:=0,
\end{gathered}
$$

$$
\phi_{k}:=\left(\phi_{k-1}+\frac{C}{\sqrt{N}} \frac{1}{\sqrt{1-h_{k}^{2}}}\right)(\bmod 2 \pi), \quad 2 \leq k \leq N-1
$$

where the constant $C$ is chosen so that successive points will have approximately the same (Euclidean) distance apart on $S^{2}$. The point set $\hat{\omega}_{N}=\left\{\left(\phi_{k}, \theta_{k}\right)\right\}_{k=1}^{N}$ is called a generalized spiral on $S^{2}$. If we choose $C=3.6$ and plot the difference $E\left(0, \hat{\omega}_{N}\right)-f(0, N)$ for $N \leq 12,000$, we get Fig. 2. This figure shows that the generalized spiral points have, for large $N$, energy that agrees with (4.1) to within $O(N)$. In fact, although $\hat{\omega}_{N}$ does not appear to solve the Shub and Smale Problem (cf. (1.3)), numerically it gives $E\left(0, \hat{\omega}_{N}\right)-f(0, N) \leq(5 / 2) \log N$ for $2 \leq N \leq 12,000$. Furthermore, from the estimate (3.2) and the computed values of $E\left(0, \hat{\omega}_{N}\right)$, we find

$$
E\left(0, \hat{\omega}_{N}\right)-\mathcal{E}(0, N) \leq 114 \log N \quad \text { for } 2 \leq N \leq 12,000 .
$$

For $-2<\alpha<2, \alpha \neq 0$, computations indicate that these same spiral points have $\alpha$-energy that agrees with (4.1) to within $O\left(N^{1-\alpha / 2}\right)$.

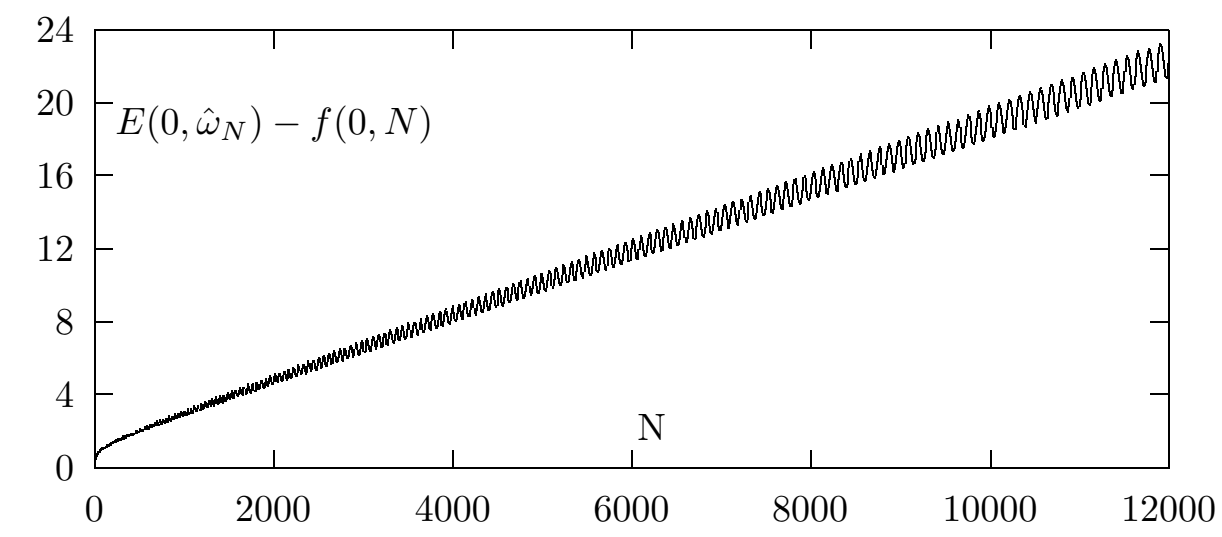

Figure 2. Behavior of Energy for Generalized Spiral 


\section{References}

1. R. Alexander. On the sum of distances between $N$ points on a sphere. Acta Math. Acad. Sci. Hungar. 23(1972), 443-448.

2. J. Beck. Some upper bounds in the theory of irregularities of distribution. Acta Arithm. 43 (1984), 115-130.

3. J. Beck and W. Chen. Irregularities of Distribution. Cambridge University Press, Cambridge, New York, New Rochelle, Melbourne, Sydney, 1987.

4. G. Björck. Distributions of positive mass which maximize a certain generalized energy integral. Ark. Mat. 3 (1956), 255-269. MR 17, 1198.

5. J. Bourgain and J. Lindenstrauss. Distribution of points on spheres and approximation by zonotopes. Israel J. of Math. (1) 64 (1988), 25-32.

6. J. Bourgain and J. Lindenstrauss. Approximation of zonoids by zonotopes. Acta Math. 162 (1989), 73-141.

7. J. H. Conway and N. J. A. Sloane. Sphere Packings, Lattices and Groups. SpringerVerlag, New York, Berlin, Heidelberg, second edition, 1993.

8. H. S. M. Coxeter. The problem of packing a number of equal nonoverlapping circles on a sphere. Trans. NY Acad. Sci. 10 (1956), 117-120. MR 18 \#356.

9. F. Robert Curl and R. E. Smalley. Fullerenes. Scientific American, 54-63, October, 1991.

10. B. E. F. Dahlberg. On the distribution of Fekete points. Duke Math. J. 45 (1978), $537-542$.

11. J. R. Edmundson. The distribution of point charges on the surface of a sphere. Acta Cryst. A48 (1992), 60-69.

12. N. D. Elkies. Personal communications.

13. T. Erber and G. M. Hockney. Equilibrium configurations of $N$ equal charges on a sphere, (letter to the editor). Journal of Physics A: Mathematical and General 24:L1369-L1377, 1991.

14. L. Fejes Tóth. On the sum of distances determined by a point set. Acta Math. Acad. Sci. Hungar. 7 (1956), 397-401. MR 21 \#5937.

15. L. Fejes Tóth. Regular Figures. Pergamon-Macmillan, New York, 1964.

16. L. Föppl. Stabile Anordnungen von Elektronen im Atom. J. Reine Angew. Math. 141 (1912), 251-301.

17. L. Glasser and A. G. Every. Energies and spacings of point charges on a sphere. Journal of Physics A: Mathematical and General 25 (1991), 2473-2482.

18. S. Lang. Introduction to Arakelov theory. Springer-Verlag, New York, 1988.

19. T. W. Melnyk, O. Knop, and W. R. Smith. Extremal arrangements of points and unit charges on a sphere: equilibrium configurations revisited. Can. J. Chem. 55 (1977), 1745-1761.

20. E. A. Rakhmanov, E. B. Saff, and Y. M. Zhou. Electrons on the sphere. In: Proceedings of Conference on Computational Methods and Function Theory, Malaysia, 1994 (to appear).

21. E. A. Rakhmanov, E. B. Saff, and Y. M. Zhou. Bounds for the product of distances between points on a sphere. (to appear).

22. M. Shub and S. Smale. Complexity of Bezout's theorem I: geometric aspects. preprint.

23. M. Shub and S. Smale. Complexity of Bezout's theorem II: volumes and probabilities. preprint.

24. M. Shub and S. Smale. Complexity of Bezout's theorem III: condition number and packing. preprint. 
25. R. E. Smalley. Great balls of carbon: the story of buckminsterfullerene. The Sciences 31 (1991), 22-28.

26. K. B. Stolarsky. Sums of distances between points on a sphere. Proc. Amer. Math. Soc. 35 (1972), 547-549.

27. K. B. Stolarsky. Sums of distances between points on a sphere. II. Proc. Amer. Math. Soc. 41 (1973), 575-582.

28. B. L. van der Waerden. Punkte auf der Kugel. Drei Zusätze. Math Ann. 125 (1952), $213-222$.

29. G. Wagner. On the product of distances to a point set on the sphere. J. Austral. Math. Soc. (Series A) 47 (1989), 466-482.

30. G. Wagner. On the means of distances on the surface of a sphere (lower bounds). Pacific J. Math. 144 (1990), 389-398.

31. G. Wagner. On the means of distances on the surface of a sphere, II (upper bounds). Pacific J. Math. 153 (1992), 381-396.

32. J. B. Weinrach, K. L. Carter, D. W. Bennett, and H. K. McDowell. Point charge approximations to a spherical charge distribution. J. Chem. Educ. 67 (1990), 995999.

33. L. L. Whyte. Unique arrangements of points on a sphere. Amer. Math. Monthly 59 (1952), 602-611.

34. B. L. Zhang, C. Z. Wang, Ho K. M., C. H. Xu, and C. T. Chan. The geometry of small fullerene cages: $C_{20}$ to $C_{70}$. Journal of Chemical Physics. 97 (1992), 50075011.

35. Y. M. Zhou. Arrangements of points on the sphere. Ph.D. thesis, University of South Florida, Tampa, FL, 1995.

E. A. Rakhmanov, Steklov Institute, 42 Vavilova St., Moscow, Russia.

E-mail address: rakhman@mph.mian.su; rakhmano@math.usf.edu.

E. B. Saff, Y. M. Zhou, Institute for Constructive Mathematics, Department of Mathematics, University of South Florida, Tampa, FL 33620.

E-mail address: esaff@math.usf.edu; zhou@math.usf.edu. 